\title{
Seeds as a Source of Plant Inhibitory Fungi: Potential for Discovering New Antibiotics
}

\author{
Benjamin J. Scherlag, PhD ${ }^{\text {* }}$, Ava Scherlag, Jack Scherlag ${ }^{2 \# \#}$ \\ ${ }^{1}$ Oklahoma University Health Sciences Center, Oklahoma City, OK, 73104 \\ ${ }^{2}$ Deer Creek School District, Edmond, Oklahoma \\ \#Middle School student, Performed as part of a summer project \\ ${ }^{\text {\#H} H i g h ~ S c h o o l ~ s t u d e n t, ~ P e r f o r m e d ~ a s ~ p a r t ~ o f ~ a ~ s u m m e r ~ p r o j e c t ~}$
}

*Corresponding author: Benjamin J. Scherlag, PhD, 1200 Everett Drive (6E103), Oklahoma City, OK. 73104, E-mail: benjamin-scherlag@ouhsc.edu

\begin{abstract}
Background: Many antibiotics have been discovered in soil, however many more have remained undetected. In the present study we used Mung bean seeds to grow plants hydroponically. Fungal infestation in some showed consistent growth inhibition.

Methods: Store bought Mung bean seeds imported from Thailand grew into mature plants in tap water $(\mathrm{pH} 7.3-7.5)$ in six beakers on top of stainless steel strainers $(20$ seeds per beaker).

Results: Of the 6 beakers containing the Mung bean plants, we compared one, showing no noticeable fungal growth (beaker 1) with two which had either moderate fungal growth (beaker 2) and one which had severe fungal infestation (beaker 3). From a quantitative perspective the wet weight/dry weight ratio of the roots cut from beaker 1 was 2.06/0.12 grams (ratio $=17.17)$ compared to those pooled from Beakers 2 and 3, 1.22/0.73 grams (ratio $=1.70, \mathrm{p}<0.05$, Chi-square analysis). The number of grown plants in the beaker 1 , 39 , was significantly greater than in the fungal groups, $22, \mathrm{p}<0.002(2 \times 2$ contingency table, Fisher exact test). Comparing the number of seeds that germinated in the Petri dishes containing tap water versus those immersed in liquid from around the roots of the fungal group found, $9 / 15$ in the former; whereas $0 / 15$ germinated in the fungal water group after a week placed in the dark, $\mathrm{p}=7 \times 10^{-4}$ (Chi-square analysis, $2 \times 2$ contingency table, Fisher exact test).
\end{abstract}

Conclusion: We found significant inhibition of plant growth and seed germination from fungi on seeds grown hydroponically.
Received Date: April 24, 2017

Accepted Date: May 3, 2017

Published Date: May 9, 2017

Citation: Scherlag, B.J., et al. Seeds as a Source of Plant Inhibitory Fungi: Potential for Discovering New Antibiotics. (2017) Lett Health Biol Sci 2(1): 61- 64.

DOI: $10.15436 / 2475-6245.17 .015$

Keywords: Mung bean seeds and plants; Hydroponic culture; Fungus; Antibiotics

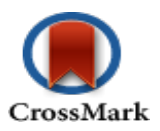

\section{Introduction}

A study in 2013 from the Center for Disease Control and Prevention ${ }^{[1]}$ reported that each year in the United States, at least 2 million people become infected with bacteria that are resistant to antibiotics and at least 23,000 people die annually as a direct result of these infections. Most of the antibiotics have been derived from the bacteria and fungi found in the soil. It has been estimated only one percent of microbes have been discovered in nature. Indeed, most pharmaceutical companies have turned to synthesizing new antibiotics, a slow and difficult means of getting these new agents to the market ${ }^{[2]}$. From the first discovery of Penicillin, a number of antibiotics have been discovered from the soil around roots of plants called the rhizosphere. The surface of the roots (rhizoplane) is highly active in terms of micro- bial activity due to root exudates which contain many different nutrients. The competition of the various microbes and fungi for these nutrients results in toxic metabolites (antibiotics) released by the fungi to survive and flourish in this environment. Thus the rhizosphere becomes an abundant source of antibiotics ${ }^{[3]}$.

The present study is based on an observation that was made while growing Mung bean plants hydroponically from store bought seeds. Some of the plant clusters developed a cottony fungus (Figure 1, Rhizoctonia solani) which markedly stunted root and stem growth and inhibited seed germination. We hypothesize that seeds from various species and sources contain residual fungi that could provide an abundant supply of potential plant and even human antibiotics, i.e., inhibitory growth factors. 


\section{Methods}

Protocol 1: Store bought Mung bean seed imported from Thailand were used in this study. Six $200 \mathrm{cc}$ beakers were filled with tap water ( $\mathrm{pH} 7.3-7.5)$. At the top of each beaker we placed a stainless steel sink strainer. Twenty Mung bean seeds were deposited in each strainer so that they were immersed in the tap water. We placed the beakers in a dark cabinet to enhance seed germination by etiolation. When the germinating beans began to show roots extending into the water below the strainer, all the beakers were placed on a counter top illuminated by fluorescent lighting. Within 10 days when the Mung beans grew into mature plants, we examined the content of the strainers to determine the presence of fungus and made comparative photographs of those with and those without noticeable fungal infestation. We then cut the roots of those with fungus and those without and blotted each group before weighing them in separate plastic containers. These were then placed in an oven at $37-40{ }^{\circ} \mathrm{C}$ for drying over a period of 2 or more days. The dried roots were weighed to determine the wet/dry weight ratios of each group. In addition, we determined the number of fully grown plants in the control group and those in the group showing fungal infestation.

Protocol 2: We placed 5 store bought Mung bean seeds in two sets of Petri dishes. Fifteen ccs of tap water $(\mathrm{pH} 7.3$ - 7.5) was added to each dish of the control group $(n=3)$; whereas the same amount of solution surrounding the roots of the plants with fungal infestation was instilled in the Petri dishes comprising the experimental group $(n=3)$. In total there were 15 seeds in each group.

\section{Statistical Analysis}

The number of grown plants in each of the control and fungal groups were compared using an unpaired T-test and analyzed using a Chi square test $(2 \times 2$ contingency table $)$ followed by a 2 tailed Fisher exact test. The same test was used to compare the number of seeds in the Petri dishes which germinated in the control group with the number of germinated seeds immersed in the solutions taken around the roots of those showing fungal infestation (fungal group). We compared the wet weight/ dry weight ratios between control and fungal group. A $p$ value of $\leq 0.05$ was considered statistically significant.

\section{Results}

Of the six beakers containing the Mung bean plants, three showed no noticeable fungal growth; two had moderate fungal growth; whereas one had severe fungal infestation (Figure 1). Figure 2 shows a qualitative comparison of three beakers, selected from the six, containing Mung bean plants two of which with different degrees of fungal infestation. Note that the water level in beaker 1 showed the lowest water level (no fungus detected) to some fungus detected (beaker 2) to seeds showing overt cottony fungus infestation (beaker 3 ). The last was associated with very little root growth compared to plants in beakers 1 and 2 . Note that beaker 1 , whose water level was the lowest, showed the greatest root growth, and greater number of grown plants compared to those containing noticeable fungus.

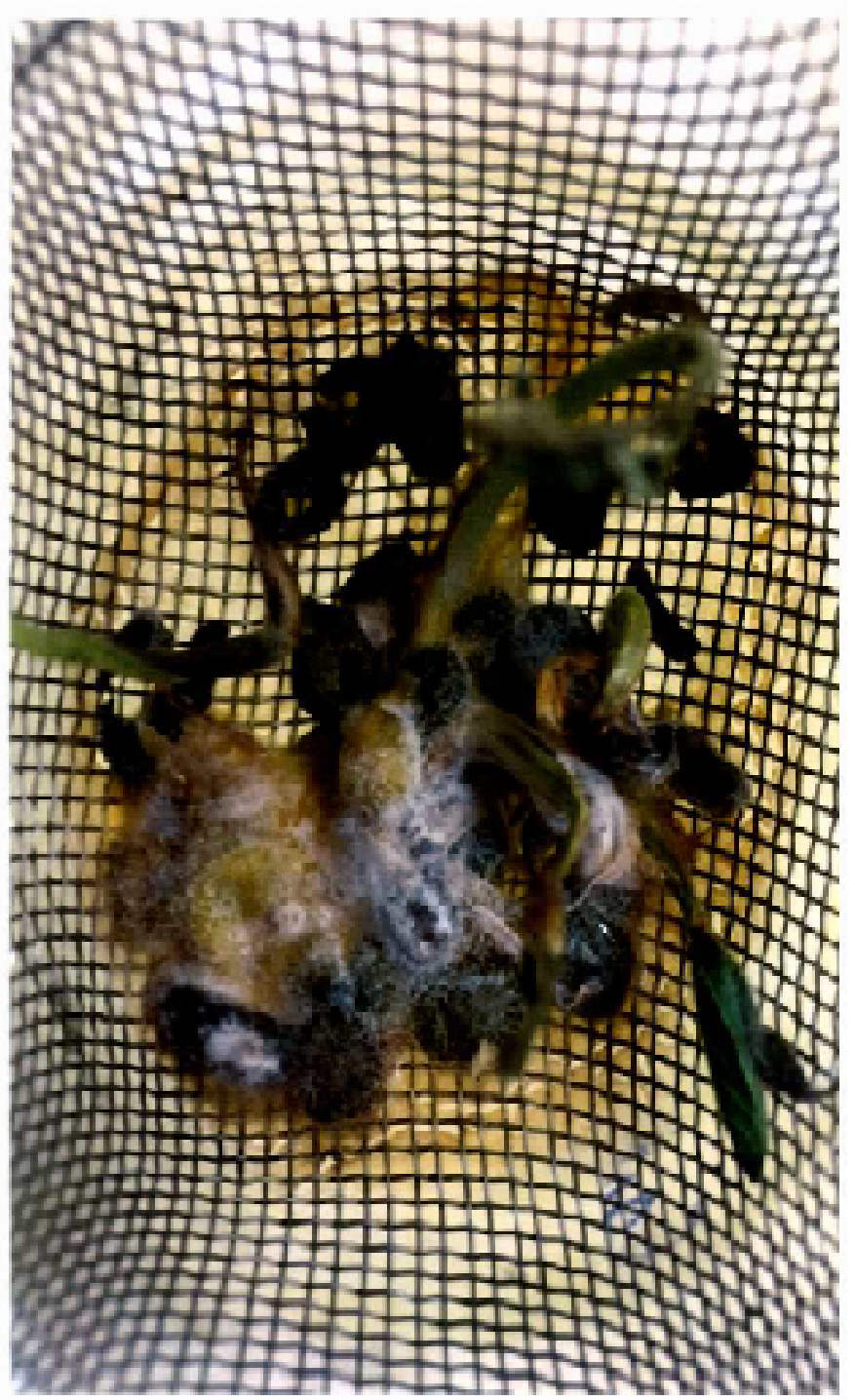

Figure 1: Germinated Mung bean seeds developed in aborted plants in a beaker with tap water $(\mathrm{pH} 7.3$ - 7.5) showing a severe infestation of the cottony fungus, Rhizoctonia solani, which inhibited root, and plant growth.

From a quantitative perspective the wet weight/dry weight ratio of the roots cut from beaker 1 was 2.06/0.12 grams $($ ratio $=17.17)$ compared to those pooled from Beakers 2 and 3 , $1.22 / 0.73$ grams $($ ratio $=1.70, \mathrm{p}=0.0004)$.

We used the Chi-square test to compare the number of mature, fully grown plants in the control group, 39 which was significantly greater than the number of grown plants in the fungal group, 22, p $<0.002(2 \times 2$ contingency table, Fisher exact test). A comparison between the number of seeds that germinated in the Petri dishes containing tap water versus those immersed in liquid from around the roots in Beaker 3 found, 9/15 in the plain water group; whereas $0 / 15$ germinated in the fungal water group after a week placed in the dark, $\mathrm{p}=7 \times 10^{-4}$ (Chi-square analysis, $2 \times 2$ contingency table, Fisher exact test).

\section{Discussion}

\section{Major Findings}

Store bought Mung bean seeds imported from Thailand were grown under hydroponic conditions. We were able to di- 
chotomize two groups, those without fungal infestation (beaker 1, Figure 2) and those with noticeable fungus (beakers 2 and 3 , Figure 2). After 10 days there was a significantly greater root growth and number of plants in beaker 1 than the pooled data from beakers 2 and 3. Also note the level of water was lowest in beaker 1 in association with the greater root, and greater number of plants corresponding to the greater water absorption in this group.

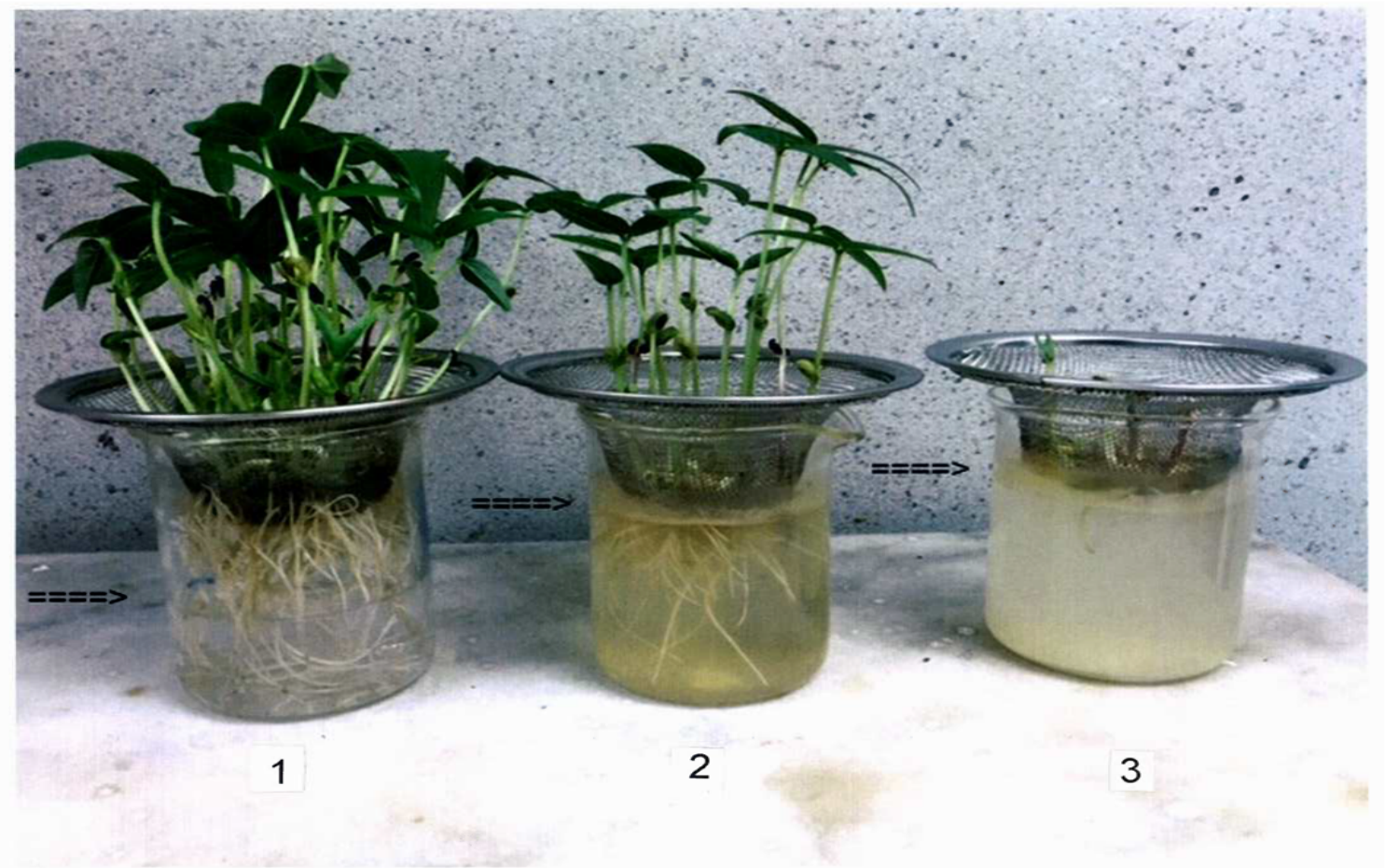

Figure 2: A comparison of three beakers, containing Mung bean plants with normal growth (beaker 1) moderate fungal infection (beaker 2) and severe fungal infestation (beaker 3). The differences in plant growth between the control group and the pooled data from the group with moderate and severe fungal infestation were quantitatively compared (Chi-square analysis, Results section).

\section{Background}

Mung beans constitute a common food source throughout Asia and parts of the Middle East. There have been several reported investigations of microflora detected in seeds of Mung beans grown in different countries. For example, Qureshi ${ }^{[3]}$ found 57 fungal isolates from soil of a variety of plants growing in several provinces of Pakistan that had antifungal activity against plant pathogens. More specifically, Agarwal et al. ${ }^{[4]}$ found six species of fungus that were isolated from 50 samples of Mung beans and Moth bean seeds. The frequency of isolation significantly varied among seed samples as was the case even with our small sample of Mung bean seeds. In a study from Saudi Arabia, 12 fungal isolates were obtained from a variety of legume or so-called pulse seeds. Of the fungal isolates, Rhizoctani solani was the most frequently found in this study and infected Mung beans with a frequency of $63 \%$, leading to a $25 \%$ decrease in seedling germination ${ }^{[5]}$. In Bangladesh, Barua et al. ${ }^{[6]}$ reported that a total of four fungi from 3 Genera were found in Mung bean samples. After subjecting these beans to a hot water treatment at $55^{\circ} \mathrm{C}$ for 15 minutes, the germination percentage of these Mung bean seeds ranged from $66-79 \%$. It is interesting to note that Mung bean seeds under hydroponic conditions in our small sample showed $60 \%$ germination. However, none of those in the fungal solution germinated within the same time period.

\section{Implications}

The greatest natural source of antibiotics comes from the soil, more specifically from the soil surrounding the roots of plants (The rhizosphere) ${ }^{[3]}$. In order to test for the presence of fungus, the seeds have to be processed by various physical, physiological and biochemical procedures which are complex and time consuming, namely the blotter incubation test, among others ${ }^{[6]}$. By growing plants hydroponically the rhizoplane now provides a direct source for fungal extraction, identification and potential discovery of new antibiotics.

\section{Limitations}

Structurally, seed bearing plants are basically composed of roots, stems and leaves. In the present report we focused our study on the effect of fungal infestation on the growth of roots, and seed germination. We did not consider leave number, leaf dimensions, etc., due to the observation that at 10 days there was both primary and secondary leaf growth. We considered the data obtained on roots, and seed germination to suffice in showing the effects of fungus derived from Mung bean seeds.

We identified the fungal contaminant of our Mung beans as Rhizoctonia solani by its characteristic appearance as a white cottony growth over the seed husks (Figure 1) and the cloudy color of the hydroponic solution seen in Figure 2 (beaker 3). We cannot rule out the presence of other fungi, however others ${ }^{[5]}$ have determined quantitatively that Rhizoctonia solani is the most frequently found of the four fungal Genera contaminating Mung bean seeds. We did not determine the fungal inhibitory effect on bacterial growth, however, in accordance with the definition of an antibiotic: "A medicine or substance, natural or synthetic that inhibits the growth or destroys plants or animals 
such as microorganisms". The fungus found in the present study falls within that definition.

\section{Conclusion}

Store bought Mung bean seeds imported from Thailand were geminated and grown hydroponically in six separate beakers. Infestation of the plant roots by the fungus, Rhizoctonia solani, was observed in 3 of the six beakers with excessive infection in one. We found significant inhibition of root growth and the number of mature plants due to fungal presence and also in seed germination. The present study suggests that it may be advantageous to extract inhibitory fungi, i.e., potential antibiotics, in hydroponically grown seeds compared to those grown in soil.

Acknowledgement: We thank Branden Pannell for his assistance in the preparation of this manuscript.

Funding Source: Supported by an unrestricted grant from the Helen and Wilton Webster Foundation through the Oklahoma Research Foundation.

Conflict of interest: The authors declare no conflict of interest.

\section{References}

1. The United States Department of Health, Center for Disease Control. (2013) Antibiotic Resistance Threats in the United States.

2. Rohn, J. The hunt is on for new antibiotics-but we have to start looking outside the lab. (2013) The Guardian US edition.

3. Qureshi, S.A. Studies on antibiotics from soil Fungi Thesis. (2003) University of Karachi, Pakistan.

4. Agarwal, T., Malhotra, A., Trivedi, P.C. Seed borne fungi of mung bean and moth bean seeds. (2012) Annals of Plant Protection Sciences 20(1): 251-252.

5. Hassan Abdullah Al-Abdalall, A. Pathological Studies of Fungi Associated with Pulse seeds during storage in Dammam Province, Kingdom of Saudi Arabia. (2008) Middle Eastern and Russian Journal of Plant Science and Biotechnology 2: 71-77.

6. Barua, J., Hossain, M.M., Rahman, A.M., et al. Control of mycoflora of farmer's stored seeds of Mung beans. (2007) Asian Journal of Plant Sciences 6: 115-121. 